\title{
Pollen morphology of selected species of Passiflora L. (Passifloraceae) from the Atlantic Forest
}

\author{
Ana Carolina Mezzonato-Pires ${ }^{1}$, Cláudia Barbieri Ferreira Mendonça ${ }^{1}$ and Vania Gonçalves-Esteves ${ }^{1 \star}$
}

Received: January 22, 2015. Accepted: April 22, 2015

\begin{abstract}
Pollen grains from twelve species of the Passifloraceae family from the Brazilian Atlantic Forest were analyzed: Passiflora subg. Astrophea (1sp.), P. subg. Decaloba (1sp.) and P. subg. Passiflora (10spp.). The current study aims to acquire additional information and feature the pollen morphology of the herein studied species to help setting a more precise taxa delimitation. Acetolyzed pollen grains were measured, described and illustrated using light and scanning electron microscopy. The pollen grains were medium or large sized, oblate spheroidal, suboblate, prolate spheroidal and subprolate; 4-colpate (P. kermesina), 6-12-pantocolporate (P. mediterranea), 6-colpate (P. mucronata), 6-colporate (P. pentagona), 12-colporate (P. misera) or 6-syncolpate (in most species). The presence of reticulate sexine, pseudopercula, pontopercula and/or opercula was observed. The endoaperture was just found in $P$. pentagona and P. misera. It was concluded that pollen morphology is an important source of taxonomic features useful for distinguishing species and characterizing the three subgenera. The current study provides additional information that, along with other previously published studies, will enable a better understanding of phylogenetic relations among these strains.
\end{abstract}

Keywords: Atlantic Forest, palynotaxonomy, Passiflora, Passifloraceae, sandbanks

\section{Introduction}

Passifloraceae s.s. holds about 17 genera and 630 species, which are widely distributed in tropical regions (Wilde 1974; Holm-Nielsen et al. 1988; Brummitt \& Powell 1992; Deginani 1999). Passiflora is its most representative genus, which presents approximately 400 taxa. The genus was initially divided into 23 subgenera (Killip 1938; Escobar 1989) and subsequently reduced to just four: Astrophea, Deidamioides, Decaloba and Passiflora.

Brazil hosts approximately 150 Passifloraceae species, 87 of them are endemic (Bernacci et al. 2014). The State of Rio de Janeiro is considered to be one of the greatest fauna and flora endemism centers in the country (Bergallo et al. 2000; Rocha et al. 2003). According to Bernacci et al. (2014), the state is represented by 41 species.

Most of the herein analyzed species (Passiflora alata, P. amethystina, $P$. edulis, $P$. farneyi, $P$. foetida, $P$. kermesina, P. mediterranea, P. misera, P. mucronata, P. pentagona, $P$. racemosa and $P$. setacea) can be found in sandbanks in the State of Rio de Janeiro. These species show wide distribution and are found in almost all Brazilian regions, except for Passiflora kermesina, $P$. pentagona and $P$. mucronata, which are restricted to the Southeast region of the country and P. farneyi, which is just found in the State of Rio de Janeiro (Bernacci et al. 2014).
The Atlantic Forest is an important "hotspot", i.e., one of the richest and most threatened biological regions in the world; its endemic rate is particularly high (Mittermeier et al. 2000). As already highlighted by Candido et al. (2013) as well as by preceding authors (Myers et al. 2000; Rocha et al. 2007), "although restinga habitats were once distributed along most all the coastline in the State of Rio de Janeiro, they have been diminishing at alarming rates due to native vegetation removal for human occupation purposes".

The main pollen studies on Passifloraceae s.s. species started to be conducted back on 1965, when Presting (1965) analyzed the morphology of pollen grains and suggested an evolutionary theory based on apertural and opercular types. Spirlet (1965) used pollen data to help taxonomic classification. Later studies such as those by García et al. (2002), Araújo \& Santos (2004), Milward-de-Azevedo et al. (2004; 2010), Barrios et al. (2005), Dettke \& Santos (2009), Evaldt et al. (2011), Tangarife et al. (2011), Mezzonato-Pires (2013) and Verdasca et al. (2013) also helped improving the palynological knowledge on the Passifloraceae s.s. family.

The current study aims to feature pollen morphology and identify informative characters that may help increasing knowledge on the genus and subgenus of this plant group. It also aims to study the phylogeny of the group and to gather helpful information to further conservation efforts.

\footnotetext{
${ }^{1}$ Laboratório de Palinologia, Museu Nacional, Universidade Federal do Rio de Janeiro, 20940-040, Rio de Janeiro, RJ, Brazil

* Corresponding author: esteves.vr@gmail.com
} 


\section{Material and methods}

The current study investigated pollen grains of 12 Passiflora species, subgenus Astrophea (1 sp.), P. subg. Decaloba (1 sp.) and P. subg. Passiflora (10 spp.). The herein used botanical material consisted of flowers in anthesis and /or flower buds picked from specimens deposited in different herbaria in the State of Rio de Janeiro; their acronyms were related according to the Index Herbariorum (Thiers, continuously updated).

Material used in the study: Passiflora alata Curtis: Brazil. Rio de Janeiro: São João da Barra, III/1939, *A. Sampaio 8175 (R); Carapebus, road to Praia da Capivara, 10/29th/1996, M.C. Oliveira 441 (R). Passiflora amethystina Mikan: Brazil. Rio de Janeiro: Teresópolis, Serra dos Órgãos National Park, between shelters 3 and 2, 04/29th/1962, *E. Santos 1235 et al. (R). Passiflora edulis Sims: Brazil. Rio de Janeiro: Guanabara Road, Recreio dos Bandeirantes, Itapeba Restinga, Lagoa das Taxas, 07/06th/1965, Newton Santos 5309 (R); Cabo Frio, Cabo Frio Restinga, 01/20th/1947, D. Sucre 1427 (RB); Maricá, Ponta Negra, 11/26th/1952, ${ }^{*}$ J. Vidal s/n (R106018). Passiflora farnyei Pessoa \& Cervi: Brazil. Rio de Janeiro: Cabo Frio, 19/11/1967, D. Sucre 1940 (R). Passiflora foetida L.: Brazil. Maranhão: Arari, camping area by Lagoa Malhada Grande, without date, J.G. Silva 2021 and J.A.F. Costa 1602 (R). Passiflora kermesina Link. \& Otto: Brazil. Rio de Janeiro: Carapebus, São Lázaro Farm, 12/07th/1994, Correia 617 et al (R). Passiflora mediterranea Vell.: Brazil. Rio de Janeiro: Morro Queimados, 01/17th/1932, *Brade 11287 (R). Passiflora misera Kunth: Brazil. Santa Catarina: BR 101, Km 348, $3 \mathrm{Km}$ north of Jaguaruna, 01/22nd/2001, ${ }^{*}$ V. J. Pott 4283 \& A. Pott (R). Passiflora mucronata Lam: Brazil. Rio de Janeiro: Macaé, Cabuinas, Lagomar Resort, Lagoa Jurubatiba, $9.3 \mathrm{Km}$ from NUPEM, Ca. $350 \mathrm{~m}$ from PNRJ entrance, 02/11th/2009, * M.F. Castilhori 262 and I.E. Santo et al (R); Rio de Janeiro, Arpoador, 03/29th/1978, P. Laclette 446 et al. (R); Carapebus, 2nd sandy line, after the road to Carapebus, 03/26th/1996, V. Esteves 928 et al. (R); Carapebus, close to Lagoa Cabuinas, 09/23rd/1997, M.C. de Oliveira 568 et al. (R). Passiflora pentagona Mast.: Brazil. Rio de Janeiro: Quissamã, ${ }^{*}$ C. Farney et al. 3420, 10/29th/1994 (RB); Espírito Santo, Itaúnas, Itaúnas State Park, Milward, M. \& Van der Vem, P.H.L. 35, 02/01st/2001 (RB). Passiflora racemosa Brot.: Brazil. Rio de Janeiro: Maricá, Morro do Macaco, 09/19th/1986, ${ }^{*} J$.G. Silva 615 et al. (R). Passiflora setacea DC.: Brazil. Rio de Janeiro: Maricá, Morro do Macaco, 09/19th/1986. ${ }^{*}$ J.G. Silva 600 et al. (R).

Standard specimens marked with an asterisk $\left(^{*}\right)$ next to the collector's name were chosen for each studied species. Three specimens were chosen for comparison to corroborate the results. The following criteria were adopted to choose the standard material: being preferably collected in the State of Rio de Janeiro and being identified by an expert on the family. The used slides were deposited in the Palynology Collection of Álvaro Xavier Moreira Palynology Laboratory, in the Botany Department at National Museum of Federal University of Rio de Janeiro.

The pollen samples to be analyzed under light microscopy were prepared according to the acetolytic method by Erdtman (1952), which was modified by Melhem et al. (2003); and measured after seven preparation days (SalgadoLabouriau 1973).

Twenty-five (25) polar diameter (PD) and equatorial diameter (ED) measurements of pollen grains in equatorial view, 10 equatorial diameter measurements and measurements of the side of the apocolpus - both in polar view (EDPV) - were performed. The grains were distributed among three slides. The statistical analysis was done by calculating the arithmetic mean $(\mathrm{x})$, sample standard deviation $(\mathrm{s})$, mean deviation $\left(\mathrm{s}_{\mathrm{x}}^{-}\right)$, the coefficient of variability $(\mathrm{CV} \%)$, the confidence interval of $95 \%$ and the variation rate. Ten (10) pollen grains were measured to set other features such as apertures and exine layers. Ten (10) similar pollen grain measurements were obtained from additional material deposited in another collection (from this point on referred to as comparison material). Such measures were taken to check the stability of data collected from the reference material (Abreu et al. 2012; 2014; Moreira et al. 2013).

The terminology herein used meets that by Barth \& Melhem (1988), Punt et al. (2007). It takes into account the size, shape and ornamental pattern of sexine. The definitions by Presting (1965) and Dettke \& Santos (2009) were applied to apertures description.

Non-acetolyzed pollen grains were assembled on stubs with double-sided carbon tape and sputter-coated (for $3 \mathrm{~min}$ ) with a thin palladium-gold layer. The photomicrographs were taken under scanning electron microscopy using the JSM-5310 microscope (JEOL, Ltd., Tokyo, Japan), in the Electron Microscopy Center of Invertebrates Department at National Museum of Federal University of Rio de Janeiro.

\section{Results}

Pollen grains subordinated to three subgenera were analyzed: P. subg. Astrophea: Passiflora pentagona (Fig. 1A-C), P. subg. Decaloba: Passiflora misera (Fig. 1D-G), and P. subg. Passiflora: Passiflora alata (Fig. 1H), Passiflora amethystina (Fig. 1I), Passiflora edulis (Fig. 1J-K), Passiflora farneyi (Fig. 1L), Passiflora foetida (Fig. 2A), Passiflora kermesina (Fig. 2B-C), Passiflora mediterranea (Fig. 2D-E), Passiflora mucronata (Fig. 2F-H), Passiflora racemosa (Fig. 2I, J) and Passiflora setacea (Fig. 2K-L).

The palynological description was arranged according to the following pollen features (Figs. 1 e 2): size; polarity; dispersal unit; size, shape, and type of apertures; opercula types; exine sculpture. The results were presented in a single description and summarized in Tables 1 to 5 . 


\section{Polarity, dispersal unit, size and shape}

Isopolar pollen grains were found in most of the species and the apolar grains were found in P. kermesina and P. mediterranea (P. subg. Passiflora) (Tabs. 2-4, Fig. 2); they were considered to be large sized in most of the species. Medium sized pollen grains were just found in P. pentagona. Just five species were analyzed in equatorial view. Their polar and equatorial diameters were measured and shapes established. Thus, the suboblate shape was found in $P$. edulis and $P$. mucronata; oblate spheroidal in $P$. alata, prolate spheroidal in $P$. misera and subprolate in $P$. pentagona. Passiflora pentagona presented the smallest polar $(48.6 \mu \mathrm{m})$ and equatorial diameters $(42.6 \mu \mathrm{m})$; whereas $P$. edulis showed the largest polar $(63.0 \mu \mathrm{m})$ and equatorial diameters $(67.5$ $\mu \mathrm{m}$ ), in equatorial view (Tab. 2). P. pentagona showed the lowest equatorial diameter, in polar view $(44.2 \mu \mathrm{m})$ and P. amethystina showed the largest one $(87.4 \mu \mathrm{m}) ;$ P. farneyi had the lowest value related to apocolpium side $(14.3 \mu \mathrm{m})$ and $P$. amethystina showed the largest one $(25.0 \mu \mathrm{m})$ (Tab. 4$)$. P. kermesina species had the lowest values in D1 and D2 (Tab. 3).

\section{Number and type of apertures}

The apertures are not easily distinguishable due to the intense ornamentation of most pollen grains. Different types of apertures were found in the studied species. P. alata, P. amethystina, P. edulis, P. farneyi, P. foetida, $P$. racemosa and $P$. setacea had 6-syncolpate apertures; $P$. kermesina, 4-colpate apertures; $P$. mediterranea pollen grains were heteromorphic, with 6-12-pantocolpate apertures; P. misera, had 12-colporate pollen grains; P. mucronata, 6-colpate apertures; $P$. pentagona, 6-colporate apertures (Tab. 1). Passiflora misera featured six slightly lolongate endoapertures (Fig. 1E) and P. pentagona, presented three single lalongate endoapertures, in each pair of ectoaperture (Fig. 1B-C, Tab. 5).

\section{Opercula}

P. alata, P. amethystina, P. edulis, P. farneyi, P. foetida, $P$. racemosa and $P$. setacea species showed three pseudopercula (Figs. 1H-J, L, 2A, I, K); P. kermesina, four pseudopercula (Fig. 2B); P. pentagona and P. mucronata, three pontopercula (Figs. 1B, C, 2F, G); P. mediterranea, six to twelve pseudopercula (Fig. 2D-E); and P. misera, six opercula (Fig. 1D, F). After acetolysis, pseudopercula detached from the murus (Fig. $1 \mathrm{H})$ in syncolpate species (P. alata, P. amethystina, $P$. edulis, $P$. farneyi, $P$. foetida, $P$ racemosa and $P$. setacea).

\section{Exine sculpture}

Reticulate exine was found in all species; $P$. misera and P. pentagona had the lowest lumina $(3.0$ e $3.6 \mu \mathrm{m})$, the other species had larger lumina $(7.3-16.3 \mu \mathrm{m})$. P. alata had the widest murus $(4.8 \mu \mathrm{m})$ and $P$. misera presented the narrowest murus $(0.9 \mu \mathrm{m})$ (Tab. 5$)$.

The scanning electron microscopy showed that muri are supported by conspicuous columellae (except in $P$. misera and $P$. pentagona). The upper part of the muri was psilate, in all studied species.

The species of the Passiflora (P. amethystina, P. edulis, $P$. farneyi, $P$. foetida, $P$. mediterranea and $P$ racemosa) subgenus had muri with greater sinuosity (Figs. 1I, K-L, 2A, D-E J); except for $P$. alata, $P$. kermesina, $P$. mucronata and $P$. setacea, which showed less sinuous muri (Figs. 1H, 2C, H, L). On the other hand, the species in the other two subgenera showed straight muri (Fig. 1B, G). Passiflora mucronata, P. misera and $P$. pentagona showed perforated muri. The lumina are just ornamented in species of the Passiflora subgenus. P. setacea showed more dense and attached bacula inside the lumina.

Exine thickness widely varied among species. The thickest exine was found in P. amethystina (exine: ca. $9.9 \mu \mathrm{m}$, sexine: ca. $5.9 \mu \mathrm{m}$ and nexine: ca. $4.0 \mu \mathrm{m})$. The thinnest exine was seen in P. misera (exine: ca. $2.4 \mu \mathrm{m}$, sexine: ca. $1.7 \mu \mathrm{m}$, nexine: ca. $0.7 \mu \mathrm{m}$ ).

\section{Pollen key to pollen grain separation in the studied Passiflora species:}

1. Apolar pollen grains

2. 4-colporate pollen grains P. kermesina (subg. Passiflora)

2. 6-12-colporate pollen grains. P. mediterranea (subg. Passiflora)

1. Isopolar pollen grains

3. Colporate pollen grains

4. Large pollen grains, 6 apertures P. misera (subg. Decaloba)

4. Medium pollen grains, 12 apertures. P. pentagona (subg. Astrophea)

3. Colpate pollen grains

5. Non-syncolpate apertures P. mucronata (subg. Passiflora)

5. Syncolpate apertures

6. Small polar area (ca. $0.29-0.33 \mu \mathrm{m}$ )

7. Granula densely together in lumen, exine thickness ca. $5.2 \mu \mathrm{m}$ P. setacea (subg. Passiflora)

7. Granula not densely together in lumen, exine thickness $\geq 6.3 \mu \mathrm{m}$

8. Exine thickness ca. $9.9 \mu \mathrm{m}$. P. amethystina (subg. Passiflora)

8. Exine thickness ca. $6.3 \mu \mathrm{m}$ P. edulis (subg. Passiflora) 
6. Very small polar area (ca. $0.21-0.25 \mu \mathrm{m})$

9. Little sinuous reticulum muri, wide (ca. $4.8 \mu \mathrm{m}$ ); exine ca. $4.9 \mu \mathrm{m}$ P. alata (subg. Passiflora)

9. Very sinuous reticulum muri, narrow (ca. 1.3-1.9 $\mu \mathrm{m}$ ); exine $\geq 6.8 \mu \mathrm{m}$

10. Exine ca. $6.8 \mu \mathrm{m}$, colpus width ca. $5.0 \mu \mathrm{m}$..

P. racemosa (subg. Passiflora)

10. Exine $\geq 7.6 \mu \mathrm{m}$, colpus width ca. $1.0-1.5 \mu \mathrm{m}$

11. Exine ca. 8.1 $\mu \mathrm{m}$, Confidence Interval 95\% of equatorial diameter in polar view $(\mathrm{EDPV})=60.9-63.0 \mu \mathrm{m}$ P. farneyi (subg. Passiflora) 11. Exine ca. $7.6 \mu \mathrm{m}$, Confidence Interval $95 \%$ of equatorial diameter in polar view $(\mathrm{EDPV})=$ $79.0-84.5 \mu \mathrm{m}$ P. foetida (subg. Passiflora)

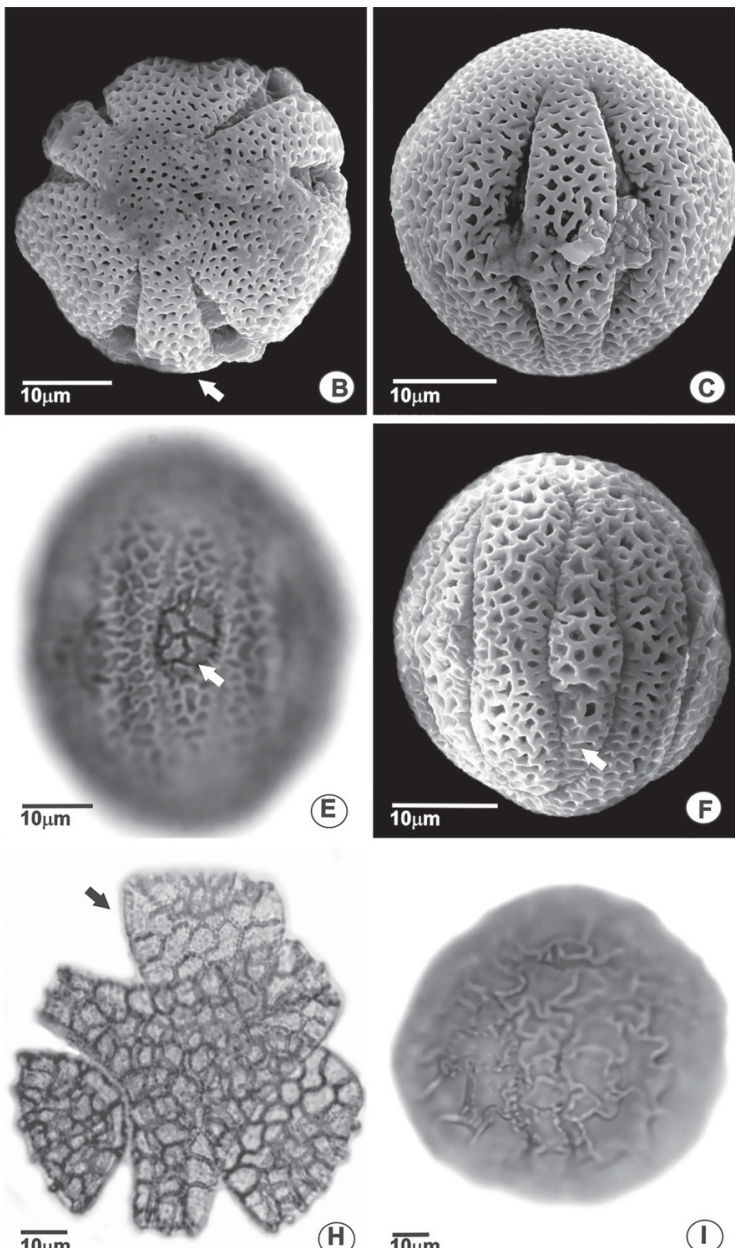

(H) $\overline{10 \mu m}$

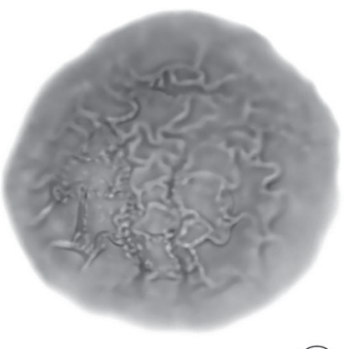

(1)
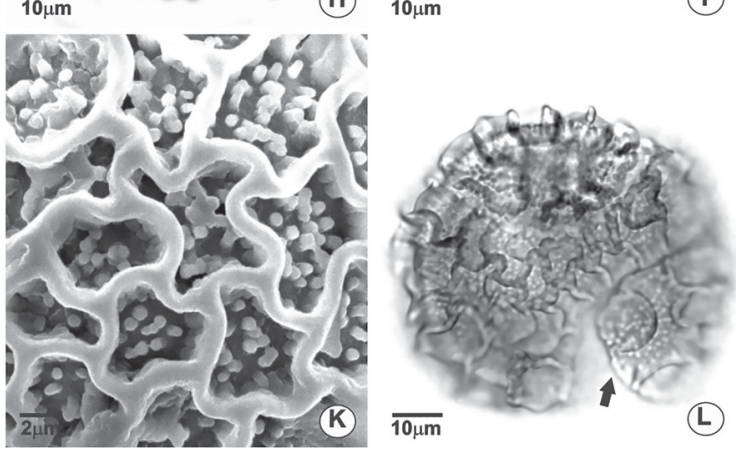

Figure 1. Photomicrographs and electron micrographs of Passiflora species pollen grains. A-C. P. pentagona - A. optical cut in polar view (LM), B. general aspect in polar view (SEM), C. general aspect in equatorial view (SEM); D-G. P. misera - D. general aspect in polar view (SEM), E. endoaperture detail (LM), F. general aspect in equatorial view (SEM), G. ornamentation detail (SEM); H. Passiflora alata Curtis - general aspect in polar view, detaching pseudopercula (LM); I. P. amethystina Mikan - general aspect in polar view (LM); J-K. P. edulis Sims - J. general aspect in polar view (SEM), K. ornamentation detail (SEM); L. P. farneyi - general aspect in polar view $(\mathrm{LM})$. Arrows: $\mathrm{B}=$ pontopercula, $\mathrm{E}=$ endoaperture, $\mathrm{F}=$ opercula, $\mathrm{H}-\mathrm{L}=$ pseudopercula. 

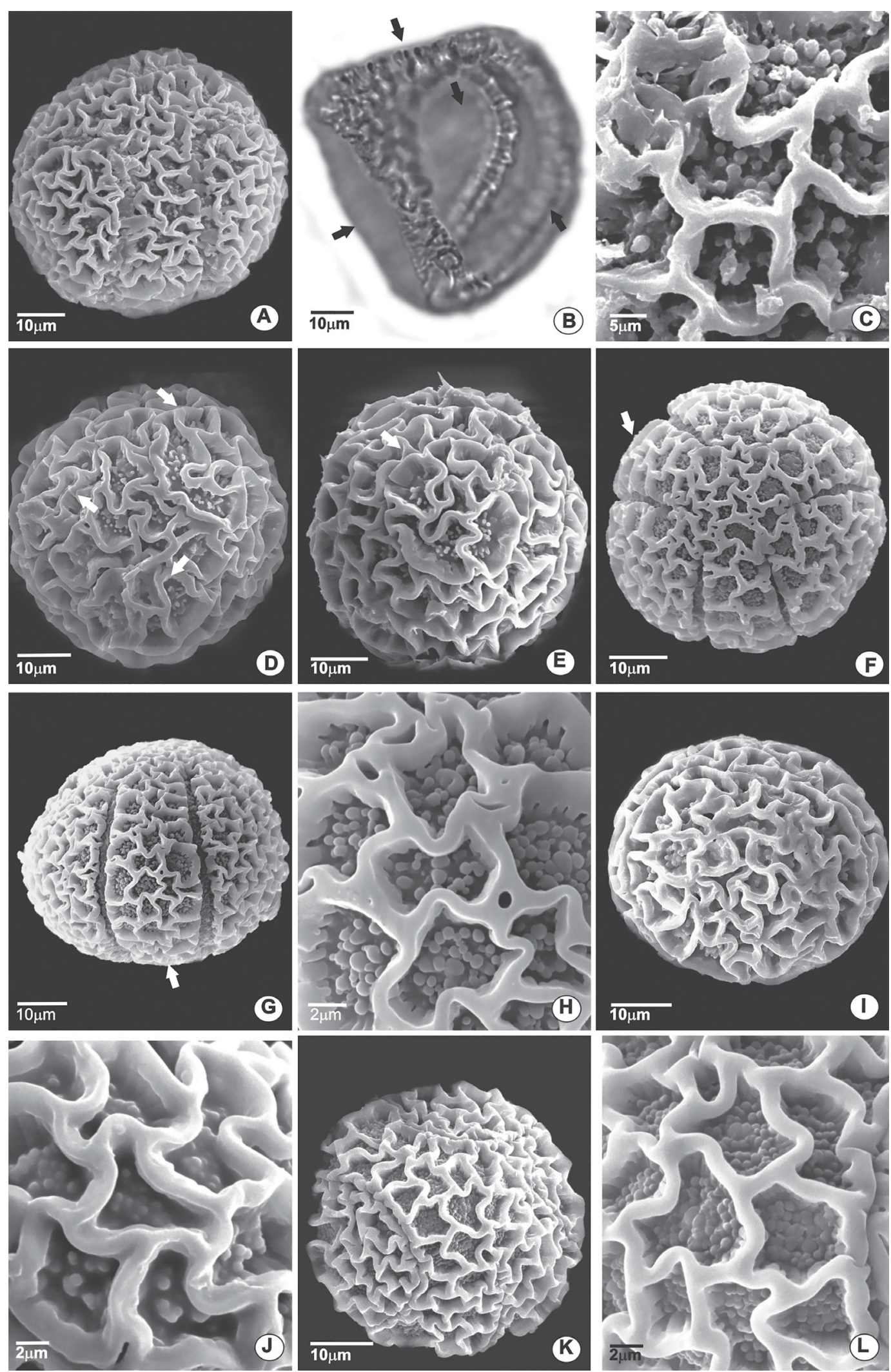

Figure 2. Photomicrographs and electron micrographs of Passiflora species pollen grains

A. P. foetida - general aspect in polar view (SEM); B-C. P. kermesina - region of the four pseudopercula (LM), B. general aspect in polar view (SEM), C. ornamentation detail (SEM); D-E. P. mediterranea - general aspect (SEM); F-H. P. mucronata - F. general aspect in polar view (SEM), G. general aspect in equatorial view (SEM), H. ornamentation detail (SEM); I-J. P. racemosa - I. general aspect in equatorial view (SEM), J. ornamentation detail (SEM); K-L. P. setacea - K. general aspect in polar view (SEM), L. ornamentation detail (SEM). Arrows: B = apertures, D, E = pseudopercula, F, G = pontopercula. 
Table 1. Morphological characterization of the analyzed Passiflora species pollen grains.

\begin{tabular}{|c|c|c|c|c|}
\hline Subgenus & Taxa & Size & Aperture & Endoaperture \\
\hline \multicolumn{5}{|l|}{ Astrophea } \\
\hline & P. pentagona & medium & 6-colporate & lalongate \\
\hline \multicolumn{5}{|l|}{ Decaloba } \\
\hline & P. misera & large & 12-colporate & slightly lolongate \\
\hline \multicolumn{5}{|l|}{ Passiflora } \\
\hline & P. alata & large & 6-sincolpate & absent \\
\hline & P. amethystina & large & 6-sincolpate & absent \\
\hline & P. edulis & large & 6-sincolpate & absent \\
\hline & P. farneyi & large & 6-sincolpate & absent \\
\hline & P. foetida & large & 6-sincolpate & absent \\
\hline & P. kermesina & large & 4-colpate & absent \\
\hline & P. mediterranea & large & 6-12-pantocolpate & absent \\
\hline & P. mucronata & large & 6-colpate & absent \\
\hline & P. racemosa & large & 6-sincolpate & absent \\
\hline & P. setacea & large & 6-sincolpate & absent \\
\hline
\end{tabular}

Table 2. Measures (in $\mu \mathrm{m}$ ) of pollen grains in equatorial view: polar diameter (PD); equatorial diameter (ED), Passiflora species ( $\mathrm{n}=25) . \mathrm{x} \pm \mathrm{s}_{\mathrm{x}}-\mathrm{-}$ arithmetic mean \pm standard deviation; $95 \% \mathrm{CI}$ - 95\% confidence interval; $\mathrm{PD} / \mathrm{ED}$ - polar:equatorial diameter ratio.

\begin{tabular}{|c|c|c|c|c|c|c|c|c|}
\hline \multirow{2}{*}{ Species } & \multicolumn{3}{|c|}{ Polar Diameter } & \multicolumn{3}{|c|}{ Equatorial Diameter } & \multirow{2}{*}{$\mathrm{PD} / \mathrm{ED}$} & \multirow{2}{*}{ Shape } \\
\hline & Range & $x- \pm s_{x-}^{-}$ & CI 95\% & Range & $x- \pm s_{x-}^{-}$ & CI 95\% & & \\
\hline P. alata & $61.5-67.5$ & $62.6 \pm 0.5$ & $61.5-63.7$ & $65.0-75.0$ & $68.9 \pm 0.6$ & $67.5-70.2$ & 0.90 & oblate spheroidal \\
\hline P. edulis & $67.5-72.5$ & $63.0 \pm 1.0$ & $60.9-65.1$ & $67.5-75.0$ & $72.1 \pm 0.7$ & $57.7-86.5$ & 0.87 & suboblate \\
\hline P. misera & $50.0-62.5$ & $56.9 \pm 0.7$ & $55.5-58.3$ & $47.5-57.5$ & $51.4 \pm 0.5$ & $50.3-52.5$ & 1.11 & prolate spheroidal \\
\hline P. mucronata & $50.0-65.0$ & $57.0 \pm 1.0$ & $55.0-59.0$ & $65.0-74.5$ & $68.8 \pm 0.6$ & $67.5-69.9$ & 0.83 & suboblate \\
\hline P. pentagona & $41.2-52.5$ & $48.6 \pm 0.5$ & $47.6-49.6$ & $37.5-47.5$ & $42.6 \pm 0.6$ & $41.4-43.8$ & 1.14 & subprolate \\
\hline
\end{tabular}

Table 3. Measures (in $\mu \mathrm{m}$ ) of diameter 1 and 2 of apolar pollen grains from Passiflora species $(\mathrm{n}=25) . \mathrm{x}_{\mathrm{x}} \mathrm{s}_{\mathrm{x}}-\mathrm{arithmetic}$ mean \pm standard deviation; $95 \%$ CI $95 \%$ confidence interval; D1/D2 - relation between diameter 1 and diameter 2.

\begin{tabular}{lcccccc}
\hline \multicolumn{1}{c}{ Species } & D1 & \multicolumn{5}{c}{ D2 } \\
\hline \\
\cline { 2 - 7 } P. kermesina & Range & x- $\pm \mathrm{s}_{\mathbf{x}}$ & CI 95\% & Range & $\mathrm{x}_{-} \pm \mathrm{s}_{\mathrm{x}}-$ & CI 95\% \\
P. mediterranea & $60.0-75.0$ & $64.5 \pm 0.6$ & $63.3-65.7$ & $60.0-75.0$ & $65.3 \pm 0.6$ & $64.1-66.5$ \\
\hline
\end{tabular}

Table 4. Measures (in $\mu \mathrm{m}$ ) of pollen grains in polar view: equatorial diameter in polar view (EDPV), side apocolpium (SA), polar area index (PAI) and exine from Passiflora species $\left(\mathrm{n}=10,{ }^{*} \mathrm{n}=25\right.$, for pollen grains which fall preferably in polar view). $\mathrm{x} \pm \mathrm{s}_{\mathrm{x}}-\mathrm{arithmetic}$ mean \pm standard deviation; $95 \%$ CI $-95 \%$ confidence interval.

\begin{tabular}{|c|c|c|c|c|c|c|c|c|c|c|}
\hline \multirow[t]{2}{*}{ Species } & \multicolumn{2}{|c|}{ EDPV } & \multirow[b]{2}{*}{ CI 95\% } & \multicolumn{2}{|c|}{ SA } & \multicolumn{2}{|c|}{ Polar area } & \multicolumn{3}{|c|}{ Exine } \\
\hline & Range & $x \pm s_{x}^{-}$ & & Range & $\mathrm{x}$ & PAI & & total & sexine & nexine \\
\hline P. alata & $62.5-72.5$ & 68.5 & - & $16.2-18.7$ & 17.3 & 0.25 & very small & 4.9 & 2.2 & 2.7 \\
\hline P. amethystina* & $77.5-90.0$ & $87.4 \pm 0.6$ & $86.2-88.6$ & $22.5-27.5$ & 25.0 & 0.29 & small & 9.9 & 5.9 & 4.0 \\
\hline P. edulis & $71.2-80.0$ & 73.6 & - & $17.5-25.0$ & 22.5 & 0.31 & small & 6.3 & 5.3 & 1.0 \\
\hline P. farneyi* & $57.5-67.5$ & $62.0 \pm 0.5$ & $60.9-63.0$ & $13.0-15.0$ & 14.3 & 0.23 & very small & 8.1 & 4.1 & 4.0 \\
\hline P. foetida* & $55.0-87.5$ & $81.8 \pm 1.3$ & $79.0-84.5$ & $19.0-23.0$ & 19.9 & 0.24 & very small & 7.6 & 4.7 & 2.9 \\
\hline P. misera & $45.0-62.5$ & 50.9 & - & $18.0-25.0$ & 20.9 & 0.41 & small & 2.4 & 1.7 & 0.7 \\
\hline P. mucronata & $42.5-50.0$ & 45.5 & - & $20.0-28.0$ & 23.1 & 0.51 & large & 8.8 & 5.8 & 3.0 \\
\hline P. pentagona & $37.5-50.0$ & 44.2 & - & $22.5-30.0$ & 24.8 & 0.56 & large & 2.8 & 1.7 & 1.1 \\
\hline P. racemosa* & $62.5-80.0$ & $73.9 \pm 0.7$ & $72.3-75.4$ & $12.0-20.0$ & 15.7 & 0.21 & very small & 6.8 & 4.4 & 2.4 \\
\hline P. setacea* & $62.5-72.5$ & $69.3 \pm 0.6$ & $68.0-70.6$ & $17.5-25.0$ & 23.1 & 0.33 & small & 5.2 & 4.1 & 1.1 \\
\hline
\end{tabular}


Table 5. Measures (in $\mu \mathrm{m}$ ) of the apertures, murus width and lumen length of Passiflora species pollen grains ( $\mathrm{n}=10)$.

\begin{tabular}{|c|c|c|c|c|c|c|}
\hline \multirow[t]{2}{*}{ Species } & \multicolumn{2}{|c|}{ Ectoaperture/Colpus } & \multicolumn{2}{|c|}{ Endoaperture } & \multirow{2}{*}{$\begin{array}{r}\text { Muri } \\
\text { width }\end{array}$} & \multirow{2}{*}{$\begin{array}{c}\text { Lumen } \\
\text { diameter }\end{array}$} \\
\hline & length & width & length & width & & \\
\hline P. alata & --- & 5.1 & --- & --- & 4.8 & 12.6 \\
\hline P. amethystina & --- & 4.0 & --- & --- & 1.9 & 14.3 \\
\hline P. edulis & --- & 1.7 & --- & --- & 2.5 & 16.3 \\
\hline P. farneyi & --- & 1.5 & --- & --- & 1.9 & 10.3 \\
\hline P. foetida & --- & 1.0 & --- & --- & 1.3 & 12.8 \\
\hline P. kermesina & --- & 5.0 & --- & --- & 1.6 & 10.3 \\
\hline P. mediterranea & --- & 1.7 & --- & --- & 2.0 & 11.9 \\
\hline P. misera & 41.0 & 1.3 & 9.9 & 8.5 & 0.9 & 3.6 \\
\hline P. mucronata & --- & 2.0 & --- & --- & 1.5 & 7.3 \\
\hline P. pentagona & 36.5 & 3.8 & 8.5 & 21.0 & 2.5 & 3.0 \\
\hline P. racemosa & --- & 5.0 & --- & --- & 1.8 & 11.5 \\
\hline P. setacea & --- & 5.0 & --- & --- & 1.0 & 10.0 \\
\hline
\end{tabular}

Table 6. Comparison of the main papers already published on Passiflora species, focusing on the apertural type.

$\mathrm{CPO}=$ colporoidate; $\mathrm{CP}=$ colporate; $\mathrm{C}=$ colpate $\mathrm{SC}=$ syncolpate; $\mathrm{GC}=$ geminicolpate; $\mathrm{PP}=$ pantoporate; $\mathrm{PC}=$ pantocolpate.

\begin{tabular}{|c|c|c|c|c|c|c|c|c|c|c|c|c|c|}
\hline Species studied & $\begin{array}{c}\text { Presting } \\
\text { (1965) }\end{array}$ & $\begin{array}{l}\text { Spirlet } \\
(1965)\end{array}$ & $\begin{array}{c}\text { Roubik } \\
\text { \& } \\
\text { Moreno } \\
(1991)\end{array}$ & $\begin{array}{l}\text { García et } \\
\text { al.. (2002) }\end{array}$ & $\begin{array}{l}\text { Mellhem et } \\
\text { al. (2003) }\end{array}$ & $\begin{array}{l}\text { Araujo } \\
\& \text { Santos } \\
(2004)\end{array}$ & $\begin{array}{c}\text { Milward- } \\
\text { de } \\
\text { Azevedo } \\
\text { (2004; } \\
2010)\end{array}$ & $\begin{array}{l}\text { Dettke } \\
\text { \& Santos } \\
(2009)\end{array}$ & $\begin{array}{l}\text { Evaldt } e t \\
\text { al. (2011) }\end{array}$ & $\begin{array}{c}\text { Tangarife } \\
\text { et al. } \\
(2011)\end{array}$ & $\begin{array}{l}\text { Mezzonato- } \\
\text { Pires (2013) }\end{array}$ & $\begin{array}{l}\text { Verdasca et } \\
\text { al. (2013) }\end{array}$ & $\begin{array}{c}\text { Present } \\
\text { study }\end{array}$ \\
\hline P. alata & - & - & - & - & $6 \mathrm{C}$ & $6 \mathrm{SC}$ & - & $6 \mathrm{C}$ & $6 \mathrm{SC}$ & - & - & $6 \mathrm{SC}$ & $6 \mathrm{SC}$ \\
\hline P. amethystina & $6 \mathrm{CPO}$ & $6 \mathrm{GC}$ & - & - & - & - & - & $6 \mathrm{C}$ & $6 \mathrm{SC}$ & - & - & - & $6 \mathrm{SC}$ \\
\hline P. edulis & $6 \mathrm{CPO}$ & $6 \mathrm{GC}$ & - & - & - & - & - & $6 \mathrm{C}$ & $6 \mathrm{SC}$ & $6 \mathrm{CPO}$ & - & $\begin{array}{c}\text { 6SC, 8-10 } \\
\text { PP }\end{array}$ & $6 \mathrm{SC}$ \\
\hline P. farneyi & - & - & & & & & - & & & - & - & - & $6 \mathrm{SC}$ \\
\hline P. foetida & $6 \mathrm{CP}$ & - & $6 \mathrm{C}$ & $6 \mathrm{C}$ & - & $6 \mathrm{SC}$ & - & - & $6 \mathrm{SC}$ & - & - & - & $6 \mathrm{SC}$ \\
\hline P. kermesina & $8 \mathrm{C}$ & - & - & - & - & - & - & - & - & - & - & - & $4 \mathrm{C}$ \\
\hline P. mediterranea & $12 \mathrm{C}$ & - & - & - & - & & - & - & - & - & - & $\begin{array}{c}\text { 66SC, } \\
8(10-12) \\
\text { PC }\end{array}$ & $6-12 \mathrm{C}$ \\
\hline P. misera & $6 \mathrm{CP}$ & - & - & $12 \mathrm{C}$ & - & $6 \mathrm{C}$ & $12 \mathrm{CP}$ & $6 \mathrm{CP}$ & $6 \mathrm{CP}$ & - & - & - & $12 \mathrm{CP}$ \\
\hline P. mucronata & $6 \mathrm{CPO}$ & - & - & - & - & - & - & - & - & - & - & - & $6 \mathrm{C}$ \\
\hline P. pentagona & - & - & - & - & - & - & - & - & - & - & $6 \mathrm{CP}$ & - & $6 \mathrm{CP}$ \\
\hline P. racemosa & - & $6 \mathrm{GC}$ & - & - & - & - & - & - & - & - & - & - & $6 \mathrm{SC}$ \\
\hline P. setacea & $8 \mathrm{C}$ & - & - & - & - & $6 \mathrm{SC}$ & - & - & - & - & - & - & $6 \mathrm{SC}$ \\
\hline
\end{tabular}

$\mathrm{CPO}=$ colporoidate $\mathrm{CP}=$ colporate $\mathrm{C}=$ colpate $; \mathrm{SC}=$ sincolpate $\mathrm{GC}=$ geminicolpate; $\mathrm{PP}=$ pantoporate; $\mathrm{PC}=$ pantocolpate

\section{Discussion}

The 12 species subordinated to the three palynologically studied subgenera could be initially separated into two major groups according to apertural type (colpate and colporate). The group with colpate aperture can be syncolpate and it coveres most of the species: apolar colpate (P. kermesina and P. mediterranea) or 6-colpate $(P$. mucronata); all species with colpate aperture belong to the Passiflora subgenus. The other group consists of two species, $P$. misera and $P$. pentagona, which belong to the Decaloba and Astrophea subgenera, respectively. P. mis- era pollen grains are 12-colporate with 6 endoapertures, whereas $P$. pentagona pollen grains are 6 -colporate with 3 endoapertures.

The exine in most of the species presented large and very ornamented lumina. P. setacea was different, because it had a bigger amount of densely distributed bacula in the lumina in comparison to other species. Passiflora mucronata stood out for the presence of perforations; a feature not found in other species from the Passiflora subgenus. The ornamentation in $P$. misera and $P$. pentagona species is similar because these species show reticula with smaller and little ornamented lumina and perforated muri. 
The herein elaborated pollen key allowed separating the species among subgenera, although some attributes were found in more than one subgenus. Passiflora subg. Passiflora showed apolar and isopolar pollen grains, besides syncolpate (one species) or non-syncolpate apertures. Passiflora subg. Astrophea and Passiflora subg. Decaloba were close in the key. However, they were separated from Passiflora subg. Passiflora, due to these species colpori-type aperture. Other important features that allowed separating the species regard details of lumen ornamentation, muri sinuosity, polar area and apertures size as well as exine thickness.

Previous studies showed great controversy in describing the types and number of apertures in genus Passiflora (see Tab. 6). Such difficulty concerned terminology (colpus, colporus, operculum types) and the level of observation (light microscope x scanning electron microscope). Some of these differences could be especially seen in older articles.

Passiflora alata was already described as having 6-colpate pollen grains (Melhem et al. 2003; Dettke \& Santos 2009) or as being 6-syncolpate (Araújo \& Santos 2004; Evaldt $e t$ al. 2011), similarly to the pollen grain described in the current paper.

Presting (1965) considered P. amethystina to be 6-colporoidate; Spirlet (1965), found it to be 6-geminicolpate; Dettke \& Santos (2009), classified it as 6-colpate; and Evaldt et al. (2011), as 6-syncolpate; as it was described in the present paper.

According to Presting (1965) and Tangarife et al. (2011), P. edulis is 6 colporoid; Spirlet (1965) classify it as 6 geminicolpi; the description by Dettke \& Santos (2009) find it to be 6-colpi; and, according to Evaldt et al. (2011) and the current results, it is 6-syncolpi.

Presting (1965) found 6 colpori in P. foetida; Roubik \& Moreno (1991) and García et al. (2002), 6-colpi; and Araújo \& Santos (2004), Evaldt et al. (2011) and the current study, classified it as 6-syncolpi.

P. kermesina and P. mucronata pollen grains were analyzed by Presting (1965) and the current study. Presting considered $P$. kermesina aperture as 8-colpate and P. mucronata, as 6-colporoidate. Results in the current study do not meet those found by Presting (1965), since he considered the presence of 4-colpi in the first species and colpus aperture type in the second specie. Presting (1965) described P. mediterranea aperture as 12-colpate; and Verdasca et al. (2013), as 6-syncolpate or 8 (10-12) pantocolporate, whereas the current study considered the aperture as 6-12-colporate.

Passiflora misera was considered by Araújo \& Santos (2004) as having 6-colpi; it was 6- colpori, according to Presting (1965), Dettke \& Santos (2009) and Evaldt et al. (2011); 12 colpi, according to García et al. (2002); and 12-colpori, according to Milward-de-Azevedo et al. $(2004 ; 2010)$. The current study corroborates the results found by the last authors.

The 6-colporate aperture type found in P. pentagona by Mezzonato-Pires (2013) was the same one found by the current study.
Passiflora racemosa pollen grains were described by Spirlet (1965) as 6-geminicolpate. The current study described it as 6-syncolpate.

Passiflora setacea was described by Presting (1965) as having 8-colpi. According to Araújo \& Santos (2004) and to the current study it had 6-syncolpi.

P. farneyi is an endemic species in the State of Rio de Janeiro (Bernacci et al. 2014), and it is not easily found in the field. Its first pollen grain analysis was done in the current study.

As it was already emphasized by other authors, it is worth analyzing pollen morphology under transmission electron microscope as well as studying its ontogeny to confirm whether or not and when aperture fusion takes place in pollen grains from this family.

As it was evidenced in previous studies, pollen morphology in the Passifloraceae family is very important for the taxonomy of the group. The family is eurypalynous and in-depth knowledge of its features will enable assessing the constituencies and the organization of its infrageneric categories, since such attributes have been completely recorded. The current study is another source of information that, along with other previously published studies, will enable better understanding the already known phylogenetic strains.

\section{Acknowledgments}

We thank Amanda Veiga (Invertebrates Department at the National Museum of the Federal University of Rio de Janeiro) for providing technical assistance in the scanning electron microscopy. This study got financial support from Conselho Nacional de Desenvolvimento Científico e Tecnológico (CNPq, National Council for Scientific and Technological Development) and from Fundação de Amparo a Pesquisa do Estado do Rio de Janeiro (FAPERJ, Foundation for the Support of Research in the State of Rio de Janeiro).

\section{References}

Abreu VHR, Bove CP, Philbrick CT, Mendonça CBF, Gonçalves-Esteves V. 2012. Pollen morphology of the aquatic Brazilian endemic genus Castelnavia Tul. \& Wedd. (Podostemaceae). Plant Systematics and Evolution 298: 1455-1461.

Abreu VHR, Mendonça CBF, Goncalves-Esteves V. 2014. Pollen morphology of selected species of the subfamily Bombacoideae (Malvaceae sensu lato). Acta Botanica Brasilica 28: 352-360.

Araújo RCMS, Santos FAR. 2004. Palinologia de espécies do Gênero Passiflora L. (Passifloraceae) da Chapada Diamantina, Bahia, Brasil. Sitientibus Série Ciências Biológicas 4: 37-42.

Barrios L, Cartano CM, Cardoso CI, Déeckenbrugge, GC, Arroyane JA, Olaya CA 2005. Caracterización Del pollen de espécies de los géneros Passiflora e Dilkea. Acta Agronômica 54: 19-23.

Barth OM, Melhem TS. 1988. Glossário ilustrado de palinologia. Campinas, Ed. Unicamp.

Bergallo HG, Rocha CFD, Alves MAS, Sluys MV. 2000. A fauna ameaçada de extinção do estado do Rio de Janeiro. Rio de Janeiro, Editora Universidade Federal do Rio de Janeiro. 
Bernacci LC, Cervi AC, Milward-de-Azevedo MA, Nunes TS, Imig DC, Mezzonato AC. 2014. Passifloraceae in Lista de Espécies da Flora do Brasil. Jardim Botânico do Rio de Janeiro. http://floradobrasil.jbrj.gov. br/jabot/floradobrasil/FB12527. 05 Dec. 2014.

Brummitt RK. 1992. Vascular plants families and genera. Kew, Royal Botanic Gardens.

Candido RS, Fourny ACS, Goncalves-Esteves V, Lopes RC. 2013. Hippeastrum species in areas of restinga in the state of Rio de Janeiro, Brazil: pollen characters. Acta Botanica Brasilica 27: 661-668.

Deginani NB. 1999. Passifloraceae L., Flora Del Valle de Lerma. Aportes Botanicos de Salta - Ser. Flora 6: 1-20.

Dettke GA, Santos RP. 2009. Tipos de aberturas dos grãos de pólen de espécies de Passiflora L. (Passifloraceae). Acta Botanica Brasilica 23: 1119-1128.

Erdtman G. 1952. Pollen morphology and plant taxonomy - Angiosperms. Stockholm, Almqvist and Wiksell.

Escobar LK. 1989. A new subgenus and five new species in Passiflora (Passifloraceae) from South America. Annals of Missouri Botanical Garden 76: 877-885.

Evaldt ACP, Bauermann SG, Cancelli RR, Acioli M, Neves PCP. 2011. Morfologia polínica de Passifloraceae Juss. ex Kunth. no Rio Grande do Sul, Brasil. Revista Brasileira de Biociências, Porto Alegre 9: 75-87.

García MTA, Galati BG, Anton AM. 2002. Microsporogenesis, microgametogenesis and pollen morphology of Passiflora spp. (Passifloraceae). Botanical Journal of the Linnean Society 139: 383-394.

Holm-Nielsen LB, Jørgensen PM, Lawesson JE. 1988. Passifloraceae. In: Harling G. Andersson L. (eds.) Flora of Ecuador. Vol 31. Göteborg \& Stockholm, University of Göteborg \& Swedish Museum of Natural History. p. 1-130.

Killip EP. 1938. The american species of Passifloraceae. Publication Field Museum of Natural History - Botanical Series 19: 1-613.

Melhem TS, Cruz-barros MAV, Corrêa AMS, Makino-Watanabe H, Silvestre-Capelato MSF, Gonçalves-Esteves V. 2003. Variabilidade polínica em plantas de Campos de Jordão (São Paulo, Brasil). Boletim do Instituto de Botânica 16: 9-104.

Mezzonato-Pires AC. 2013. Palinotaxonomia de Passiflora L. subgênero Astrophea (DC.) Masters (Passifloraceae). Msc. Thesis, Museu Nacional/Universidade Federal do Rio de Janeiro, Brazil.

Milward-de-Azevedo MA, Gonçalves-Esteves VL, Baumgratz JFA. 2004. Palinotaxonomia das espécies de Passiflora L. subg. Decaloba (DC.) Rchb. (Passifloraceae) no Sudeste do Brasil. Revista Brasileira de Botânica 27: 655-665.
Milward-de-Azevedo MA, Souza FC, Baumgratz JFA, Gonçalves-Esteves V. 2010. Palinotaxonomia de Passiflora L. subg. Decaloba (DC.) Rchb. (Passifloraceae) no Brasil. Acta Botanica Brasilica 24: 133-145.

Mittermeier RA, Myers N, Gil PR, Mittermeier CG. 2000. Hotspots: Earth's biologically richest and most endangered terrestrial ecorregions. Mexico City, CEMEX.

Moreira FF, Vaz AMSF, Mendonça CBF, Goncalves-Esteves V. 2013. The systematic value of pollen morphology in trees and shrubs species of Bauhinia L. (Caesalpinioideae subg. Bauhinia - sect. Pauletia) occurring in Brazil. Acta Botanica Brasilica 27: 400-417.

Myers N, Mittermeier RA, Mittermeier CG, Fonseca GA, Kent J. 2000. Biodiversity hotspots for conservation priorities. Nature 403: 853-858.

Presting D. 1965. Zur Morfhologie der pollenkörner der Passifloraceen. Pollen et Spores 7: 193-247.

Punt W, Blackmore S, Nilsson S, LE Thomas A. 2007. Glossary of pollen and spore terminology. Review of Paleobotany and Palynology 143: 1-81.

Rocha CFD, Bergallo HG, Alves MAS, Sluys M. 2003. A Biodiversidade nos Grandes Remanescentes Florestais do Estado do Rio de Janeiro e nas Restingas da Mata Atlântica. São Carlos, RiMa.

Rocha CFD, Bergallo HG, Van Sluys M, Alves MAS, Jamel CE. 2007. The remnants of restinga habitats in the Brazilian Atlantic Forest of Rio de Janeiro state, Brazil: habitat loss and risk of disappearance. Brazilian Journal of Biology 67: 263-273.

Roubik DW, Moreno JE. 1991. Pollen and Spores of Barro Colorado Island. Missouri Botanical Garden.

Salgado-Labouriau ML. 1973. Contribuição à Palinologia dos Cerrados. Rio de Janeiro, Academia Brasileira de Ciências.

Spirlet ML. 1965. Utilisation taxonomique dês grains de pollen de Passifloracées I. Pollen et Spores 7: 249-301.

Tangarife MMM, Caetano CM, Chávez-Servia J. 2011. Palinología de algunas especies de Passifloraceae neotropicales. Revista Investigaciones Universidade Quíndio. Armenia-Colombia 22: 141-149.

Thiers B. 2014. [continuously updated]. Index Herbariorum: a global directory of public herbaria and associated staff. New York Botanical Garden's Virtual Herbarium. http://sweetgum.nybg.org/ih/. 15 Oct. 2014.

Verdasca S, Corrêa AMS, Cruz-Barros MAV. 2013. Flora polínica da reserva do Parque Estadual das Fontes do Ipiranga (São Paulo, Brasil) Família: 54-Passifloraceae. Hoehnea 40: 727-736.

Wilde WJJO. 1974. Account of Efulensia (Passifloraceae). Blumea 22: 31-35. 